\title{
Packed bed compression visualisation and flow simulation using an erosion-dilation approach
}

T.F. Johnson ${ }^{A}$, F. lacoviello ${ }^{B}$, D.J. Hayden ${ }^{C}$, J.H. Welsh ${ }^{C}$, P.R. Levison ${ }^{C}$, P.R. Shearing ${ }^{B}$ and D.G. Bracewell ${ }^{*}$

A: Department of Biochemical Engineering, University College London, Bernard Katz, London, United Kingdom, WC1E 6BT.

B: Electrochemical Innovation Lab, Department of Chemical Engineering, University College London, Torrington Place, London, United Kingdom, WC1E 7JE.

C: Pall Biotech, 5 Harbourgate Business Park, Southampton Road, Portsmouth, United Kingdom, PO6 4BQ.

*: Author to whom correspondence should be addressed.

Key words: X-ray computed tomography, packed bed, compression, imaging, structure

\section{Abstract}

X-ray computed tomography has been demonstrated to be capable of imaging $1 \mathrm{~mL}(5 \mathrm{~mm}$ diameter, $50 \mathrm{~mm}$ height) chromatography packed beds under compression, visualising the 3D structure and measuring changes to geometry of the packing. $1 \mathrm{~mL}$ pre-packed columns did not exhibit any structural changes at vendor specified flow rate limits, however cellulose beds did compress at higher flow rates, which was imaged before, during and after flow. This was used to visualise and quantitate changes to porosity, tortuosity and permeability based on simulation of flow through the packed bed structure using the imaging data. When using a high flow rate it was found that a decrease in porosity could be measured during compression before reverting after flow had ceased, with corresponding changes to tortuosity and permeability also occurring.

X-ray CT imaging of packed beds and individual beads exposed to foulant-rich process streams resulted in considerable image quality loss, associated with residual biological material. In order to address this, digital processing using an erosion-dilation method was applied at bead and bed scales to computationally alter the porosity by adding or removing material from the existing surface to calculate the impact upon tortuosity factor. The eroded 
and dilated bead volumes of agarose, cellulose and ceramic materials were used to simulate diffusivity whilst mimicking internal bead pore constriction and blocking mechanisms.

\section{Introduction}

Two common industrial issues for chromatography systems are investigated here: compression and fouling using a 3D imaging approach in order to measure changes in geometry at both packed bed and individual bead scales. Image acquisition of chromatography materials undergoing these processes to compare to the normal state was desired, where gathering 3D datasets on actual beads and beds would enable consideration of the impact of these industrially relevant processing phenomena to flow and mass transfer characteristics.

Packed bed resins for the separation of biological products are typically made of soft materials such as agarose and cellulose, therefore compression during chromatography can occur due to several factors: excessive flow rates applied through a column [1], accumulation of particulates that cause blockages and pressure increases and a combination of these issues. Compression can be reversible where often the bed structure reverts after processing has occurred [2] although in extreme cases the compression is irreversible and can lead to column collapse which effectively makes the resin unusable, requiring replacement [3].

This is of particular relevance to industrial scale chromatography, where large column diameters up to 2 metres provide little frictional support via wall effects [4]-[6], potentially leading to the loss of a bed full of expensive sorbent [7], [8]. Feed streams in such processes often contain complex impurities from harvest and upstream operations which increases the likelihood of bed compression and collapse due to fouling [1], [9], [10], highlighting the need for better understanding of mechanistic impacts on bed structure.

Fouling of chromatography systems occurs due to the input of poorly clarified feeds and an insufficient cleaning regime between cycles to remove this material [7], [11], [12]. Coating of the surface of beads reduces the capability for desired binding species in the mobile phase to transfer within the bead, resulting in performance loss which is furthered by foulant impregnation throughout the internal structure. This can be considered as either particulate foulants, impermeable soluble foulants or permeable yet unremoved soluble foulants, however 
the purpose of this study was not to individually identify these and so are referred to as 'foulants' to encompass all credible types [13], [14].

Electron microscopy has been commonly used to image foulant accumulation due to the high resolution capabilities [15]-[18] although without sectioning using an approach such as microtomy, focused ion beam or serial block face SEM [19]-[23] is incapable of visualising the internal structure. Confocal Laser Scanning Microscopy (CLSM) [24]-[26] has been effective for investigating the presence of foulants on the surface and within chromatography beads and additionally can be used to label different components of interest [27], [28] such as nucleotides and proteins, however lacks the capability to resolve the detailed internal structure of these materials. In order to successfully visualise and quantify internal bead changes due to foulant impregnation, an approach would be required that was capable of sectioning a bead with sufficient resolution. 3D imaging of columns using MRI when considering flow between the spheres has been performed with flow analysis [29] and monoliths, an alternative to conventional liquid chromatography for downstream processing has been reconstructed into a 3D volume [30] based on 2D images from serial block face scanning electron microscopy. These high-resolution imaging approaches enhance understanding as to how structure relates to function and performance and enable positional-based analysis to determine changes throughout the geometry of the material.

In previous studies [20], [31], X-ray Computed Tomography (CT) [32]-[35] was used to visualise the 3D structure of $1 \mathrm{~mL}$ pre-packed beds and individual beads in a non-destructive manner required by electron microscopy counterparts, where porosity, tortuosity, surface area to volume ratio and average pore diameters were evaluated for commonly used materials. This tomographic approach enabled measurement of geometric discrepancies between bed structure at the column edge to the centre as well as visual identification of structural heterogeneity.

Therefore X-ray CT has been applied here to investigate the impact structural changes to prepacked beds and individual beads with respective consideration of compression and fouling effects to be imaged, where key characteristics such as porosity and tortuosity were measured as a result of changes to structure at both scales. Simulating permeability [36] of mobile phase through real column structure and diffusivity of BSA (bovine serum albumin) within a bead at the tens of nanometres scale by using the geometry acquired from X-ray CT was also performed to compare with established literature values from non-tomographic approaches. 


\section{Materials and methods}

\subsection{Packed bed compression imaging}

$1 \mathrm{~mL}$ (5 mm diameter, $50 \mathrm{~mm}$ height) pre-packed beds were supplied by Pall Biotech (Portsmouth, UK) in the form of MEP (mercapto-ethyl-pyridine) HyperCel® (cellulose matrix) and Ceramic HyperD® F columns. X-ray CT Scanning was performed using a Nikon (Tring, United Kingdom) XT H 225 ST system using setups identified in a previous study [31].. Common settings used for both sample types included 3,142 projections at 4 frames whilst applying 'reduced ring artefacts' with a 1 second exposure time and $20 \mathrm{~W}$ power, imaging in reflection mode. Ceramic imaging was performed using a tungsten target with a primary accelerating voltage of $140 \mathrm{KeV}$, whilst cellulose samples required a silver target at $100 \mathrm{KeV}$.

Columns were held in place using a Danaher Tool Group (NC, USA) $20.3 \mathrm{~mm}$ drill chuck, with tubing attached to a $20 \%$ ethanol supply in water and a peristaltic pump that was used to apply flow during experiments, where scans were 5 hours each for high resolution radiographs. Scans began after the bed settled under compression, determined to be 10 minutes after flow started in each case with an ÄKTA Pure (GE Healthcare) used to determine the pressure at set flow rates. $20 \%$ ethanol was selected as the mobile phase because it was the constituent buffer upon which previous image optimisation was performed for each material [31] and so implementing an additional change to the system beyond bed compression was avoided, although the viscosity of $20 \%$ ethanol may not be considered to be representative of foulantrich feeds. During ethanol flow, the packed bed was required to settle at a period of 5 minutes before imaging was commenced, with the same requirement for corresponding scans of the reverted bed. As with the previous study [31], scans were reconstructed into VGI and VOL files using Nikon's X-Tec 3D Reconstructor before insertion into Avizo® 9.5 (FEl, Bordeaux, France) for processing and characterisation.

\subsection{Processing and characterisation}


Both packed beds were imaged here at a $3 \mu \mathrm{m}$ pixel size across the $5 \mathrm{~mm}$ internal diameter at the top of the bed and for the individual bead volume date from a previous study [20] with a pixel size of $32 \mathrm{~nm}$ and volume dimensions of $6.4 \mu \mathrm{m}$ were loaded into Avizo Fire 9.5 (Bordeaux, France) for processing steps. The material phase was segmented from the void or buffer, with noise and artefacts also removed at this stage to provide a representative 3D volume. Geometric porosity, tortuosity and pore diameters were calculated in Avizo, with the XLab extension used to simulate permeability and diffusivity and packed bed and individual bead scales respectively on sub-volumes. Permeability through packed beds was based on measured pressure drops at 300 and $3,000 \mathrm{~cm} \cdot \mathrm{h}^{-1}$ respectively, with the properties of $20 \%$ ethanol used for the mobile phase used as inputs for the simulation.

Permeability computational experiments using the Avizo extension XLab isolates a binarised volume in a manner than prevents flow outside of the selected region aside from a respective inlet and outlet plane that have specified associated pressures whereby flow is available across the entire plane. Darcy's law is applied to the hermetically sealed box that also considers the mobile phase viscosity and is under the assumption of a Newtonian, incompressible fluid. From these iterative simulations then an absolute permeability value in $\mu \mathrm{m}^{2}$ is obtained which was normalised between states in the results and discussion section.

For digital erosion-dilation on original imaged volumes, fully processed cellulose sub-volumes at the bed scale were isolated for investigation whilst for individual bead internal structures from a previous study [20] were used; being agarose, cellulose and ceramic acquired at a 32 $\mathrm{nm}$ pixel size. Erosion and dilation commands were used in Avizo to iteratively remove or add a layer of material to the original 3D surface respectively in order to produce a series of volumes at differing porosities from the original 3 samples whilst conserving geometry. Unchanged, eroded and dilated volumes at both scales were inserted in the MATLAB® plugin TauFactor as detailed by Cooper et al. [37] to determine tortuosity factor changes due to porosity alteration of the same original volume in each case. Diffusivity simulations were used at the individual bead scale using Avizo XLab suite for original and dilated volumes, with $1 \mathrm{~g} . \mathrm{L}^{-}$ ${ }^{1}$ of BSA with a bulk diffusivity of $6 \times 10^{-11} \mathrm{~m}^{2} \cdot \mathrm{s}^{-1}$ used in each case. Computational diffusivity experiments in the Avizo extension XLab on a binarised volume to calculate molecular diffusivity of the mobile phase on a non-diffusive solid phase are considered. Fick's diffusional equations are transformed into vector-based problems solved using the 3D volume that is sealed in 4 planes by entire solid with an input and output plane left open with corresponding mobile phase concentrations inputted into the extension. 


\section{Results and discussion}

\subsection{Compression of packed beds}

Individual radiographs were used to determine the degree of packed bed height decrease for cellulose and ceramic $1 \mathrm{~mL}$ pre-packed beds between the recommended $300 \mathrm{~cm} \cdot \mathrm{h}^{-1}$ up to and beyond the linear pressure-flow relationship region [6] that would not be suitable for operation. Whilst ceramic bed heights were not found to change at any flow rate investigated and demonstrating that resin material selection can be of importance in cases where high flow rates may be desired or required, Figure 1 displays reductions in cellulose bed height during and after flow in addition to the pressure-flow relationship for the pre-packed bed.

\section{Figure 1}

It was observed that at $300 \mathrm{~cm} \cdot \mathrm{h}^{-1}$ no changes to bed height were observed, however at higher flow rates and corresponding pressure drop the bed height did reduce, with the majority of height loss occurring within 10 seconds. Notably at these flow rates above those recommended by the vendor, the cellulose packed bed did not fully revert to the original height at the top of the chamber after 10 seconds, or a further 10 minutes, resulting in a change of structure to the bed when compared to the unused column. This irreversible compression can eventually lead to bed collapse, particularly in larger diameter industrial columns which is undesirable and would require resin replacement.

The use of $20 \%$ ethanol in water as the mobile phase was due to it being the constituent buffer in the $1 \mathrm{~mL}$ pre-packed columns during imaging optimisation in a previous study [31] and so was preferred as to not introduce further change beyond movement of liquid and beads. Changing the buffer from $20 \%$ ethanol was found to reduce image quality considerably and would likely require imaging re-optimisation, however investigation into the impact of other mobile phases with different characteristics such as viscosity would be of interest to better mimic a foulant rich feed. Irreversible compression experienced by a cellulose column at a $10,000 \mathrm{~cm} \cdot \mathrm{h}^{-1}$ flow rate was imaged a week after flow had ceased, with the top of the bed displayed in Figure 2. This long term compressed state was not found to change height 
beyond the 10 seconds after release, indicating that at these higher flow rates the reverted height appears to be permanent. The high resolution required (the best spot size and therefore viable pixel size achievable in reflection mode) resulted in the axial field of view being limited, where ideally the entire length of the chamber could be viewed at the best resolution available. In order to image the entire column, tens of individual scans at 5 hours each would have to be performed, taking months for a single column and would require cycling of the column 'during' compression that may cause unrepresentative change and so imaging the entire bed was deemed unfeasible [31].

\section{Figure 2}

In Figure 2 at an extremely high flow rate of $10,000 \mathrm{~cm} \cdot \mathrm{h}^{-1}$ a $1 \mathrm{~mm}$ gap was observed between the top of the packed bed and the entrance moulding that would have been occupied by the bed before use. This demonstrated that high flow conditions, albeit entirely unrepresentative of typical use, could cause permanent changes to packed bed due to the excessive force exerted on the structure that could be visualised using X-ray CT in sufficient detail to resolve individual beads. Figure 3 displays a 2D slice through the top of the packed bed before, during and after $3,000 \mathrm{~cm} \cdot \mathrm{h}^{-1}$ flow of $20 \%$ ethanol.

\section{Figure 3}

The overall morphology of the top of the packed bed was observed to change from the geometry of the polypropylene moulding during compression and then did not fully revert into the same configuration after ethanol flow, suggesting that bead positions had moved, altering inter-bead pores that influence available flow paths. After digital processing, quantitative analysis of key structural attributes was performed to determine the impact of bed height changes due to compression on geometric characteristics before, during and after compression, with results displayed in Figure 4 measured using the top $2 \mathrm{~mm}$ of the bed. It was noted that during flow the signal to noise ratio and general quality of imaging had reduced and so more extensive measures were required to correct this for final segmentation and analysis; considering that obtaining the most accurate images in the first place is preferable for producing representative measurements. 


\section{Figure 4}

Average inter-bead porosity was found to decrease during compression before reverting afterwards, with corresponding decreases in relative permeability and increases in tortuosity. This suggested that the visual changes to bed structure observed using X-ray CT did quantitatively impact inter-bead geometry to the extent that parameters investigated here relating to flow were found to change whilst using relatively high flow rates, particularly when considering the top of the packed bed. These results also suggested that the majority of the compression was occurring near the chamber entrance, with the porosity loss in the top $2 \mathrm{~mm}$ undergoing an estimated $5 \%$ porosity reduction, where it would be $1 \%$ if compression resulted in uniform porosity loss axially.

Quantitative differences between column edge and centre were detected, as has been found in various other studies [4], [31], [38], [39] that will be more prominent in small diameter columns such as these but are insufficient in providing wall support in larger, manufacturing scale columns that are susceptible to compression. Whilst there were obvious changes to measured values 'during' compressive flow, it was of interest that there were slight changes observed in variance between 'before' and 'after' measurements. This was attributed to the reverted beads within the bed not resettling in exactly the same location as can be viewed in Figure 3 and so the inter-bead structure had shifted despite having the same amount of porosity.

The application of permeability simulations through the packed bed structure also enabled visualisation of the flow paths and relative velocities of the mobile phase through the interbead pores. It was found that the variance increased 'after' compression at both the edge and centre of the bed that also displayed discrepancies in permeability values. This was attributed to the uncontrolled resettling effect could cause areas of increased restriction or conversely small vacuous channels to form that may result in a greater degree of bed heterogeneity in terms of paths available for fluid flow despite similar averages being measured.

For pre-packed columns manual adjustment is not feasible, however for manually packed beds tests such as acetone pulses can be used to ensure that there are sufficient theoretical plates to indicate that the bed is packed to a suitable standard and will not compress dramatically under typical operation conditions. If compression persists and is impacting performance or reducing column lifetime at the required flow rates then other bead materials should be considered, for example ceramics that were not found to compress when imaged even at the extreme flow rates studied here. 
Figure 5 shows the packed bed structure at the top of the column before and during compressive flow resulting in bed compression, where permeability simulations were performed. It can be observed that the flow paths are going around the spherical beads, however they are considerably more numerous in the uncompressed case, corresponding to the drop in permeability during compression where there are fewer and more constricted networks for the mobile phase to traverse. These simulations did not account for any interaction between phases as the beads are considered as incompressible solids. It demonstrated that data from experiments can be used to improve the fidelity of simulation work based on 3D geometries of imaged materials such as packed beds and individual beads having undergone operational stresses such as elevated flow rates.

\section{Figure 5}

Alterations to bed structure would be undesirable in terms of process robustness and consistency, particularly for larger columns found in manufacturing environments that have relatively reduced wall support and could ultimately lead to irreversible compression. The surface area to volume ratio identified for these columns was found to be at the level of wellpacked beds as in a previous study [31] and so insufficient packing was not considered an issue, particularly given that no changes to bed structure were observed at the recommended flow rate. Overall, changes to inter-bead voids were possible to visualise and quantify using a tomographic approach such as X-ray CT when considering commercially available pre-packed beds, with results for each characteristic investigated displayed in Table 1.

\section{Table 1}

\subsection{Fouling and erosion-dilation}

Imaging of fouled packed beds and individual beads was found to be ineffective when using $\mathrm{X}$-ray $\mathrm{CT}$ at both scales, as the presence of protein and other associated material in a sample 
resulted in considerable loss of contrast and image quality that made it difficult to accurately represent 3D chromatographic structure. Figure 6 displays an example where packed bed image contrast is entirely lost when material is inserted into the column, whereby the original quality could be restored by cleaning the column with conventional CIP reagents. Whilst this prevented imaging and subsequent analysis of geometric aspects at both scales in the presence of foulants, clean samples have previously been successfully investigated [20], [31] and so were used as a basis for digital alteration.

\section{Figure 6}

Therefore an erosion-dilation [40] approach was taken to modify existing volumes at both scales by iteratively decreasing or increasing the material phase by 1 pixel respectively. The resulting change of porosity to the original volume causes porous inter or intra-bead channels to be altered whilst conserving overall geometry, mimicking effects such as reduction of interbead pores at the bed scale or foulant impregnation within a bead. Figure 7 displays two cellulose packed bed sub-volumes in heavy erosion-dilation conditions, where clear differences in voidage can be observed despite originating from the same $\mathrm{X}$-ray CT images that demonstrates the overall mechanism at extreme porosity values to visually demonstrate the effect; with tortuosity factor [37] that simulates diffusive flux through the generated volume determined for each eroded and dilated sample. Presenting tortuosity results for very high or low porosity data-sets was deemed to be irrelevant, as the tomographic structures obtained would not be representative of actual packed bed structure when erosion or dilation was taken beyond representative values.

\section{Figure 7}

As the material phase was dilated, porosity decreased which constricted mobile phase channels and also led to more geometric blockages that overall resulted in expected tortuosity factor increases. Likewise the opposite effects were observed when eroding the material phase that increased porosity and enabled geometric phenomena such as channelling to be possible in the packed bed structure, reducing tortuosity factor to near-1 values. Whilst for 
packed beds the change in state between before, during and after fluid flow could be visualised and quantified from a real column, the inability to achieve this for fouled beads meant that approximations by using digital alteration were necessary in this case, with Figure 8 displaying results of the erosion-dilation on three different materials. The porosity range over which erosion-dilation had to be performed in order to obtain the same number of data points was greater for individual bead structure, however this was deemed acceptable because intrabead porosity was expected to have a larger feasible range [18].

\section{Figure 8}

Simulated tortuosity factor [37] increased with dilated variants, where fewer total erosiondilation iterations from the original volume could be performed due to the geometric properties of a thin strand-like structure compared to packed bed spheres. Decreasing porosity to varying degrees was considered to be analogous to approximating two potential states that an impregnated bead could be in: material coated in a thin layer of foulant or more extensive fouling resulting in pores clogged with material, with a combination of the two also likely [18].

Relationships between intra-bead porosity and tortuosity factor were found to be very similar for all three materials of construction. This indicated that despite having visually different structures that can be considered as heterogeneous yet constant, in particular ceramic beads to agarose and cellulose may not considerably impact tortuosity when normalised against porosity, which would require manufacture of beads at specific porosities for scanning without using a relatively rapid erosion-dilation approach. The trend when considering a packed bed was different to within a bead, attributed to the mobile phase having to go around large spheres rather than through a more fibrous or strand-like structure.

Diffusion of $1 \mathrm{~g} \cdot \mathrm{L}^{-1}$ BSA was simulated through new agarose, cellulose and ceramic bead subvolumes investigated in a previous study [20] based on $32 \mathrm{~nm}$ pixel size X-ray CT imaging. Table 2 displays results for diffusivity coefficients obtained that were used to mimic foulant penetration with minor and major porosity reductions, with $32 \mathrm{~nm}$ of material phase added to the material phase in each dilation where extreme iterations would result in entire pore loss.

\section{Table 2}


It was found that the original volumes were within an order of magnitude of values stated in literature using a non-tomographic approach, with porosities also in the correct ranges. This indicated that the original images were of sufficient accuracy in representing protein diffusion through a bead, suggesting that the $32 \mathrm{~nm}$ pixel size was sufficient for this purpose. However, consideration should be made with the mAb adjusted results for ceramic which were found to be approximately an order of magnitude lower than the corresponding literature value at the low and medium porosities that have been found for this material in a previous study [20]. Previous discussion regarding the required pixel size was made where there is a large impact on values such as pore size but not tortuosity [20] and the degree of fine structure that needs to be represented to accurately perform measurements and simulations.

When increasing the pixel size by a factor of two for each material, an 8 fold increase in diffusivity on average was found, whereby doubling the pixel size results in 8 times fewer pixels per volume. Given that the $32 \mathrm{~nm}$ pixel size resulted in values the same order of magnitude as literature results, it would have been interesting to further investigate the impact of improving the pixel size again, however this was not feasible with the equipment available although an alternative approach such as focused ion beam or serial block face microtomy may be suited for this purpose. For all samples, a higher porosity resulted in an increased diffusivity coefficient measurement.

\section{Conclusions}

X-ray CT enabled the visualisation and subsequent quantification of changes to packed bed structure due to compression, which has previously been difficult to do with commercially available columns. By being capable of imaging the inter-bead space before, during and after a range of flow rates, the impact of compression could be characterised in terms of important geometric factors such as porosity, tortuosity and simulated permeability could be used to further understanding of changes to column structure.

The demonstration of using tomographic volumes for analysis based upon both raw seed data collection and the use of digital alteration such as erosion-dilation here allows for structural differences to be compared on real geometry when raw image acquisition is infeasible or difficult, such was the case with fouled columns here. This provides a basis for investigations that use $3 D$ structural information from real chromatography materials where hypothetical 
changes to geometry can be made by digitally manipulating 3D datasets to alter porosity and pore size, negating the need to produce and image many original samples. Erosion-dilation here enabled evaluation and comparison between different materials, with intra-particle diffusivity simulations of particular interest when comparing with experimentally obtained literature values and between different erosion-dilation states.

\section{Acknowledgements}

This research was supported by the UK Engineering and Physical Sciences Research Council (EPSRC) grant EP/L01520X/1. Paul Shearing acknowledges support from the Royal Academy of Engineering. We would like to thank Pall Biotech, Portsmouth, United Kingdom, for the supply and expertise concerning cellulose and ceramic chromatography materials.

\section{References}

[1] R. N. Keener, E. J. Fernandez, J. E. Maneval, and R. A. Hart, "Advancement in the modeling of pressure-flow for the guidance of development and scale-up of commercial-scale biopharmaceutical chromatography," J. Chromatogr. A, vol. 1190, no. 1-2, pp. 127-140, 2008. 10.1016/j.chroma.2008.02.113.

[2] M. Sarker and G. Guichon, "Study of the operating conditions of axial compression columns for preparative chromatography," vol. 709, pp. 227-239, 1995.

10.1016/0021-9673(95)00474-2.

[3] D. Hekmat et al., "Macroscopic investigation of the transient hydrodynamic memory behavior of preparative packed chromatography beds," J. Chromatogr. A, vol. 1218, no. 7, pp. 944-950, 2011. 10.1016/j.chroma.2010.12.092.

[4] R. A. Shalliker, B. S. Broyles, and G. Guiochon, "Physical evidence of two wall effects in liquid chromatography," J. Chromatogr. A, vol. 888, no. 1-2, pp. 1-12, 2000. 10.1016/S0021-9673(00)00517-3.

[5] R. Tran, J. R. Joseph, A. Sinclair, D. Bracewell, Y. Zhou, and N. J. Titchener-hooker, "A Framework for the Prediction of Scale-Up When Using Compressible Chromatographic Packings," 2007. 10.1021/bp060303i.

[6] J. J. Stickel and A. Fotopoulos, "Pressure - Flow Relationships for Packed Beds of Compressible Chromatography Media at Laboratory and Production Scale †," pp. 744-751, 2001. 10.1021/bp010060o.

[7] C. Jiang, J. Liu, M. Rubacha, and A. A. Shukla, "A mechanistic study of Protein A chromatography resin lifetime," J. Chromatogr. A, vol. 1216, no. 31, pp. 5849-5855, 
2009. 10.1016/j.chroma.2009.06.013.

[8] D. E. Cherrak, M. Al-bokari, E. C. Drumm, and G. Guiochon, "Behavior of packing materials in axially compressed chromatographic columns," J. Chromatogr. A, vol. 943, no. 2001, pp. 15-31, 2010. 10.1016/S0021-9673(01)01432-7.

[9] R. N. Keener, J. E. Maneval, K. C. E. Östergren, and E. J. Fernandez, "Mechanical deformation of compressible chromatographic columns," Biotechnol. Prog., vol. 18, no. 3, pp. 587-596, 2002. 10.1021/bp020051v.

[10] D. Hekmat, M. Kuhn, V. Meinhardt, and D. Weuster-Botz, "Modeling of transient flow through a viscoelastic preparative chromatography packing.," Biotechnol. Prog., vol. 29, no. 4, pp. 958-67, 2013. 10.1002/btpr.1768.

[11] A. Grönberg, M. Eriksson, M. Ersoy, and H. J. Johansson, "A tool for increasing the lifetime of chromatography resins," MAbs, vol. 3, no. 2, pp. 195-205, 2011. 10.4161/mabs.3.2.14874.

[12] L. Yang, J. D. Harding, A. V. Ivanov, N. Ramasubramanyan, and D. D. Dong, "Effect of cleaning agents and additives on Protein A ligand degradation and chromatography performance," J. Chromatogr. A, vol. 1385, pp. 63-68, 2015.

10.1016/j.chroma.2015.01.068.

[13] S. C. Siu, H. Baldascini, D. C. Hearle, M. Hoare, and N. J. Titchener-Hooker, "Effect of fouling on the capacity and breakthrough characteristics of a packed bed ion exchange chromatography column," Bioprocess Biosyst. Eng., vol. 28, no. 6, pp. 405414, 2006. 10.1016/j.chroma.2015.01.068.

[14] T. Iskra, G. R. Bolton, J. L. Coffman, and R. Godavarti, "The effect of protein a cycle number on the performance and lifetime of an anion exchange polishing step," Biotechnol. Bioeng., vol. 110, no. 4, pp. 1142-1152, 2013. 10.1002/bit.24781.

[15] E. J. Close, J. R. Salm, T. Iskra, E. Sørensen, and D. G. Bracewell, "Fouling of an anion exchange chromatography operation in a monoclonal antibody process:

Visualization and kinetic studies," Biotechnol. Bioeng., vol. 110, no. 9, pp. 2425-2435, 2013. 10.1002/bit.24781.

[16] J. M. Angelo, A. Cvetkovic, R. Gantier, and A. M. Lenhoff, "Characterization of crosslinked cellulosic ion-exchange adsorbents: 1. Structural properties," J. Chromatogr. A, vol. 1319, pp. 46-56, 2013. 10.1016/j.chroma.2013.10.003.

[17] B. D. Bowes, H. Koku, K. J. Czymmek, and A. M. Lenhoff, "Protein adsorption and transport in dextran-modified ion-exchange media. I: Adsorption," J. Chromatogr. A, vol. 1216, no. 45, pp. 7774-7784, 2009. 10.1016/j.chroma.2009.09.014.

[18] M. Pathak and A. S. Rathore, "Mechanistic understanding of fouling of protein $A$ chromatography resin," J. Chromatogr. A, vol. 1459, pp. 78-88, 2016. 10.1016/j.chroma.2016.06.084.

[19] A. Zankel, J. Wagner, and P. Poelt, "Serial sectioning methods for 3D investigations in materials science," Micron, vol. 62, pp. 66-78, 2014. 10.1016/j.micron.2014.03.002.

[20] T. F. Johnson et al., "Three dimensional characterisation of chromatography bead internal structure using $X$-ray computed tomography and focused ion beam microscopy," J. Chromatogr. A, vol. 1566, pp. 79-88, 2018. 10.1016/j.chroma.2018.06.054.

[21] W. Denk and H. Horstmann, "Serial block-face scanning electron microscopy to reconstruct three-dimensional tissue nanostructure," PLoS Biol., vol. 2, no. 11, 2004. 10.1371/journal.pbio.0020329.

[22] H. Koku, R. S. Maier, K. J. Czymmek, M. R. Schure, and A. M. Lenhoff, "Modeling of flow in a polymeric chromatographic monolith," J. Chromatogr. A, vol. 1218, no. 22, pp. 3466-3475, 2011. 10.1016/j.chroma.2011.03.064. 
[23] T. Müllner, A. Zankel, A. Höltzel, F. Svec, and U. Tallarek, "Morphological Properties of Methacrylate-Based Polymer Monoliths: From Gel Porosity to Macroscopic Inhomogeneities," Langmuir, vol. 33, no. 9, pp. 2205-2214, 2017. 10.1021/acs.langmuir.7b00337.

[24] M. S. Shapiro, S. J. Haswell, G. J. Lye, and D. G. Bracewell, "Design and characterization of a microfluidic packed bed system for protein breakthrough and dynamic binding capacity determination," Biotechnol. Prog., vol. 25, no. 1, pp. 277285, 2009. 10.1021/bp.99.

[25] S. Gerontas, M. S. Shapiro, and D. G. Bracewell, "Chromatography modelling to describe protein adsorption at bead level," J. Chromatogr. A, vol. 1284, pp. 44-52, 2013. 10.1021/bp.99.

[26] L. E. Blue et al., "Recent advances in capillary ultrahigh pressure liquid chromatography," J. Chromatogr. A, vol. 1523, pp. 17-39, 2017. 10.1016/j.chroma.2017.05.039.

[27] S. C. Siu et al., "Visualising fouling of a chromatographic matrix using confocal scanning laser microscopy.," Biotechnol. Bioeng., vol. 95, no. 4, pp. 714-23, Nov. 2006. 10.1002/bit.21028.

[28] J. Jin, S. Chhatre, N. J. Titchener-Hooker, and D. G. Bracewell, "Evaluation of the impact of lipid fouling during the chromatographic purification of virus-like particles from Saccharomyces cerevisiae," J. Chem. Technol. Biotechnol., no. June 2009, 2009. 10.1002/jctb.2290.

[29] M. H. Sankey, D. J. Holland, A. J. Sederman, and L. F. Gladden, "Magnetic resonance velocity imaging of liquid and gas two-phase flow in packed beds," $J$. Magn. Reson., vol. 196, no. 2, pp. 142-148, 2009. 10.1016/j.jmr.2008.10.021.

[30] T. Müllner et al., "Reconstruction and characterization of a polymer-based monolithic stationary phase using serial block-face scanning electron microscopy," Langmuir, vol. 28, no. 49, pp. 16733-16737, 2012. 10.1021/la3038395.

[31] T. F. Johnson, P. R. Levison, P. R. Shearing, and D. G. Bracewell, "X-ray computed tomography of packed bed chromatography columns for three dimensional imaging and analysis," J. Chromatogr. A, vol. 1487, pp. 108-115, 2017. 10.1016/j.chroma.2017.01.013.

[32] P. R. Shearing, L. E. Howard, P. S. Jørgensen, N. P. Brandon, and S. J. Harris, "Characterization of the 3-dimensional microstructure of a graphite negative electrode from a Li-ion battery," Electrochem. commun., vol. 12, no. 3, pp. 374-377, 2010. 10.1016/j.elecom.2009.12.038.

[33] F. Tariq et al., "Three-dimensional high resolution X-ray imaging and quantification of lithium ion battery mesocarbon microbead anodes," J. Power Sources, vol. 248, pp. 1014-1020, 2014. 10.1016/j.jpowsour.2013.08.147.

[34] J. R. Izzo et al., "Nondestructive Reconstruction and Analysis of SOFC Anodes Using X-ray Computed Tomography at Sub-50 nm Resolution," J. Electrochem. Soc., vol. 155, no. 5, p. B504, 2008. 10.1149/1.2895067.

[35] P. R. Shearing, J. Gelb, and N. P. Brandon, "X-ray nano computerised tomography of SOFC electrodes using a focused ion beam sample-preparation technique," J. Eur. Ceram. Soc., vol. 30, no. 8, pp. 1809-1814, 2010. 10.1016/j.jeurceramsoc.2010.02.004.

[36] F. Dolamore, C. Fee, and S. Dimartino, "Modelling ordered packed beds of spheres: The importance of bed orientation and the influence of tortuosity on dispersion," $J$. Chromatogr. A, vol. 1532, pp. 150-160, 2018. 10.1016/j.chroma.2017.12.004.

[37] S. J. Cooper, A. Bertei, P. R. Shearing, J. A. Kilner, and N. P. Brandon, "TauFactor: 
An open-source application for calculating tortuosity factors from tomographic data," SoftwareX, vol. 5, pp. 203-210, 2016. 10.1016/j.softx.2016.09.002.

[38] A. E. Reising, S. Schlabach, V. Baranau, D. Stoeckel, and U. Tallarek, "Analysis of packing microstructure and wall effects in a narrow-bore ultrahigh pressure liquid chromatography column using focused ion-beam scanning electron microscopy," $J$. Chromatogr. A, vol. 1513, pp. 172-182, 2017. 10.1016/j.chroma.2017.07.049.

[39] F. Gritti, "On the relationship between radial structure heterogeneities and efficiency of chromatographic columns," J. Chromatogr. A, vol. 1533, pp. 112-126, 2018. 10.1016/j.chroma.2017.12.030.

[40] X. Lu et al., "Multi-length scale tomography for the determination and optimization of the effective microstructural properties in novel hierarchical solid oxide fuel cell anodes," J. Power Sources, vol. 367, pp. 177-186, 2017. 10.1016/j.jpowsour.2017.09.017.

[41] H. Kempe, P. Persson, A. Axelsson, B. Nilsson, and G. Zacchi, "Determination of Diffusion Coefficients of Proteins in Stationary Phases by Frontal Chromatography," 2005. 10.1002/bit.20738. 10.1002/bit.20738.

[42] K.-F. Du, M. Yan, Q.-Y. Wang, and H. Song, "Preparation and characterization of novel macroporous cellulose beads regenerated from ionic liquid for fast chromatography.," J. Chromatogr. A, vol. 1217, no. 8, pp. 1298-304, Feb. 2010. 10.1016/j.chroma.2009.12.037. 10.1016/j.chroma.2009.12.037.

[43] J. Vajda, W. Conze, and E. Müller, "Kinetic plots in aqueous size exclusion chromatography of monoclonal antibodies and virus particles," J. Chromatogr. A, vol. 1426, pp. 118-125, 2015. 10.1016/j.chroma.2015.11.057.

10.1016/j.chroma.2015.11.057.

\section{List of Tables}

Table 1: Geometric properties of cellulose packed beds under compression. Results are reported to 3 significant figures for the top $2 \mathrm{~mm}$ of the packed bed, presenting the mean and one standard deviation for 300 and $3,000 \mathrm{~cm} \cdot \mathrm{h}^{-1}$ flow. Measurements were made based on the inter-bead pore network, considering the beads to be solid spheres and thus negating the intra-bead porous space. Note that permeability has been normalised against the 'Before edge' for each flow rate respectively which was designated as the volume up to $250 \mu \mathrm{m}$ from the wall with the rest being classified as the centre. Permeability simulations in XLAB were based off the pressure drops at the respective flow rates and properties of the $20 \%$ ethanol mobile phase. Analysis was performed on one column with average and standard deviation calculations based on sub-volume division across the top of the packed bed. 
Table 2: Diffusivity coefficient results for 3 chromatography bead materials. $6.4 \mu \mathrm{m}$ dimensions of sub-volume at $32 \mathrm{~nm}$ pixel sizes were used in each case, with a bulk diffusivity of the liquid phase being $6 \times 10^{-11} \mathrm{~m}^{2} . \mathrm{s}^{-1}$. Different porosities were generated using the erosion/dilation approach to approximate filter coating or pore blocking due to foulant impregnation. *Result reported for MAb not BSA, molecule being approximately 3 times larger but control runs indicated no change to diffusivity coefficient.

\section{List of Figures}

Figure 1: Reduction in cellulose bed height due to ethanol flow at flow rates between 0 and $11,000 \mathrm{~cm} \cdot \mathrm{h}^{-1}$. Measurements in bed height change were made 10 seconds and 10 minutes after applying flow, with reversion after flow imaged and pressure drop detected.

Figure 2: 3D render of irreversible compression of a cellulose column after $10,000 \mathrm{~cm} \cdot \mathrm{h}^{-1} \mathrm{flow}$ for 5 hours. Image acquisition was performed a week after flow was ceased.

Figure 3: Entrance to a cellulose packed bed before, during and after ethanol flow at 3,000 $\mathrm{cm} . \mathrm{h}^{-1}$. Top: Before flow application. Middle: During flow. Bottom: After flow. A red reference line has been added in each case to give an indication of bed height changes.

Figure 4: Values obtained from cellulose packed bed structure entrance. A: Geometric porosity. B: Geometric tortuosity. C: Relative permeability. Note that permeability has been normalised against the lowest value in each case. 'During' refers to the imaging taken when the bed stopped moving under compressive flow, determined to be 10 minutes after flow began. Permeability simulations in XLAB were based off the pressure drops at the respective flow rates and properties of the $20 \%$ ethanol mobile phase. Edge of column was defined as $250 \mu \mathrm{m}$ from the column edge with the rest being classified as the centre with measurements made on the top $2 \mathrm{~mm}$ of the bed. Analysis was performed on one column with average and standard deviation calculations based on sub-volume division across the top of the bed. 
Figure 5: Visualisation examples of flow simulation and compression. A: Cellulose packed bed sub-volume before compression permeability simulation. B: Permeability simulation under identical conditions in the same location, imaged during $3,000 \mathrm{~cm} \cdot \mathrm{h}^{-1}$ flow compression. Simulations were performed in Avizo XLAB using identical conditions based on pressure drop results obtained during imaging of ethanol flow.

Figure 6: Visual demonstration of image contrast loss on used columns. A: Horizontal 2D slice through an unused packed bed. B: Horizontal 2D slice through the same packed bed location after insertion of a rich IgG feed. Effective clean in place was found to restore image quality once the material in the column was removed.

Figure 7: Tortuosity factor as a result of erosion-dilation of a packed bed. A: Cellulose subvolume after extreme erosion (inter-bead porosity of $75 \%$ ). B: Cellulose sub-volume after extreme dilation (inter-bead porosity of $19 \%$ ). C: Tortuosity factor compared to porosity of each volume. Blue represents material phase in each case.

Figure 8: Tortuosity factor as a result of erosion-dilation of an individual bead sub-volume. A: Agarose sub-volume after erosion (intra-bead porosity of $80 \%$ ). B: Agarose sub-volume after extreme dilation (intra-bead porosity of $57 \%$ ). C: Tortuosity factor compared to porosity of each volume. Blue represents material phase in each case. 
Figure 1

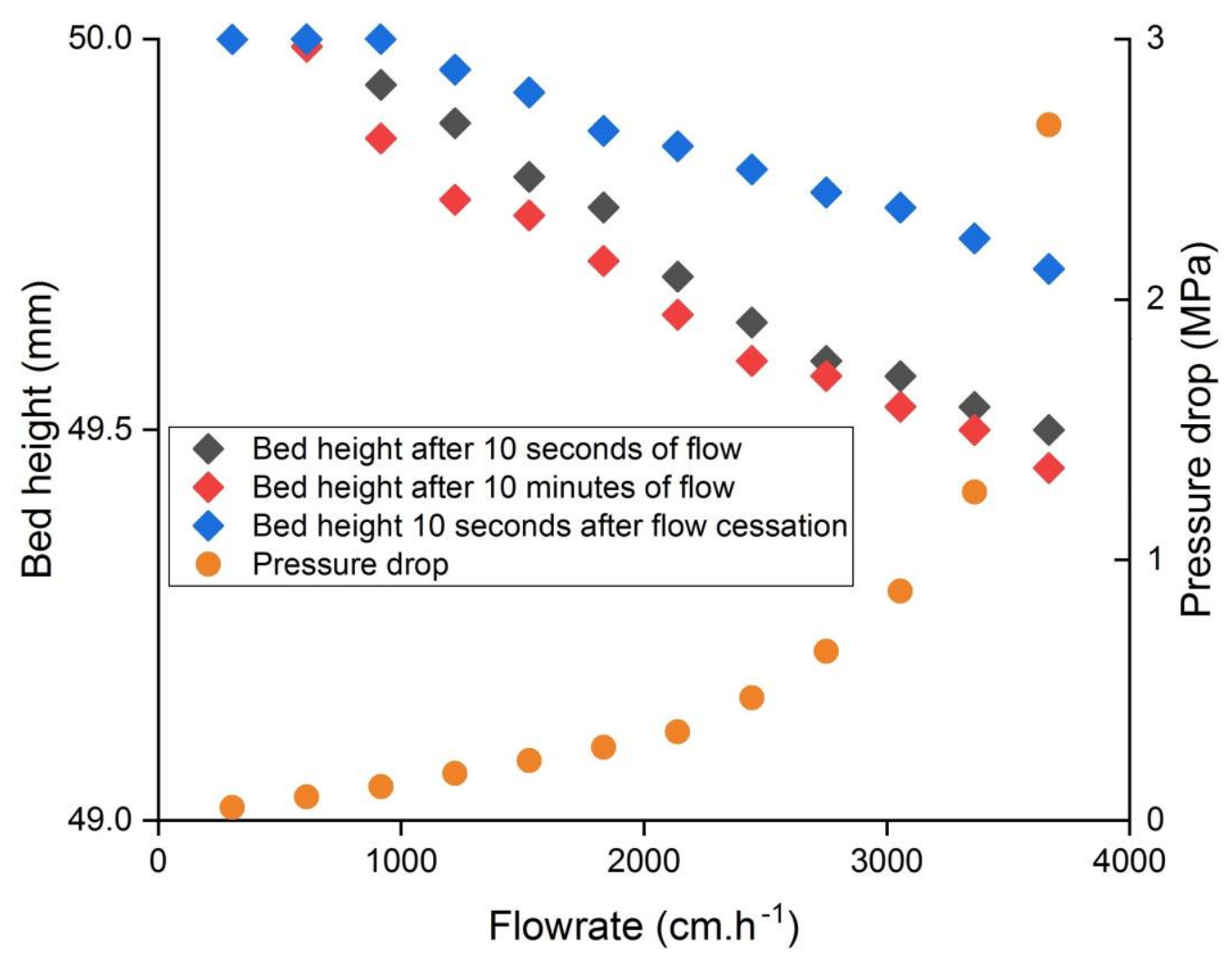


Figure 2

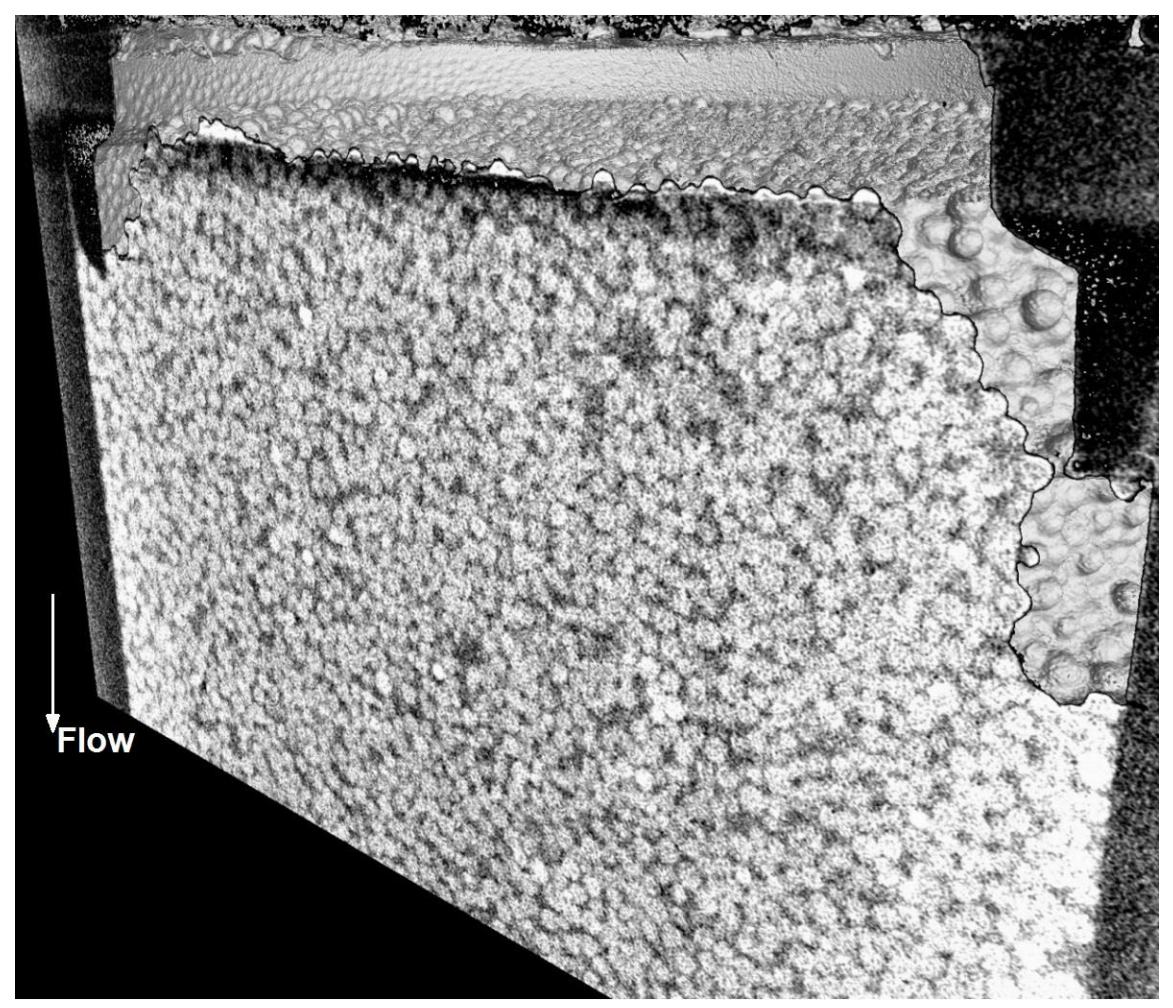

Figure 3

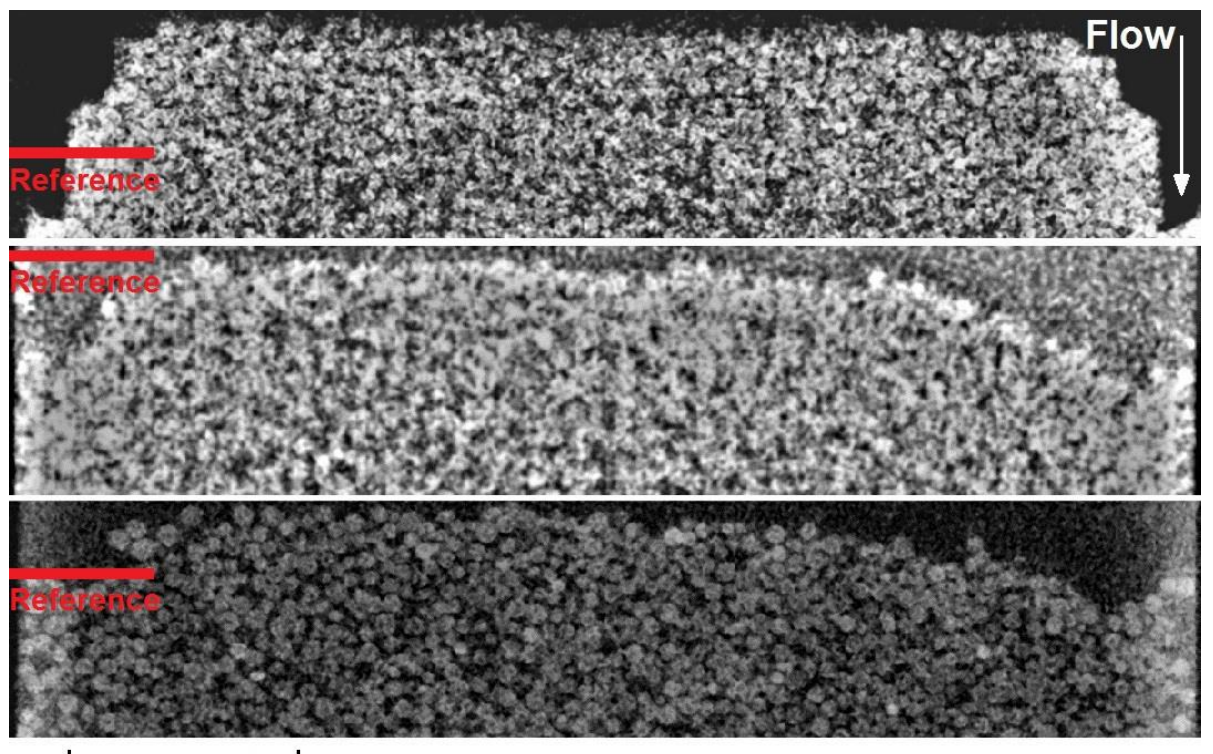

$1 \mathrm{~mm}$ 
Figure 4

A

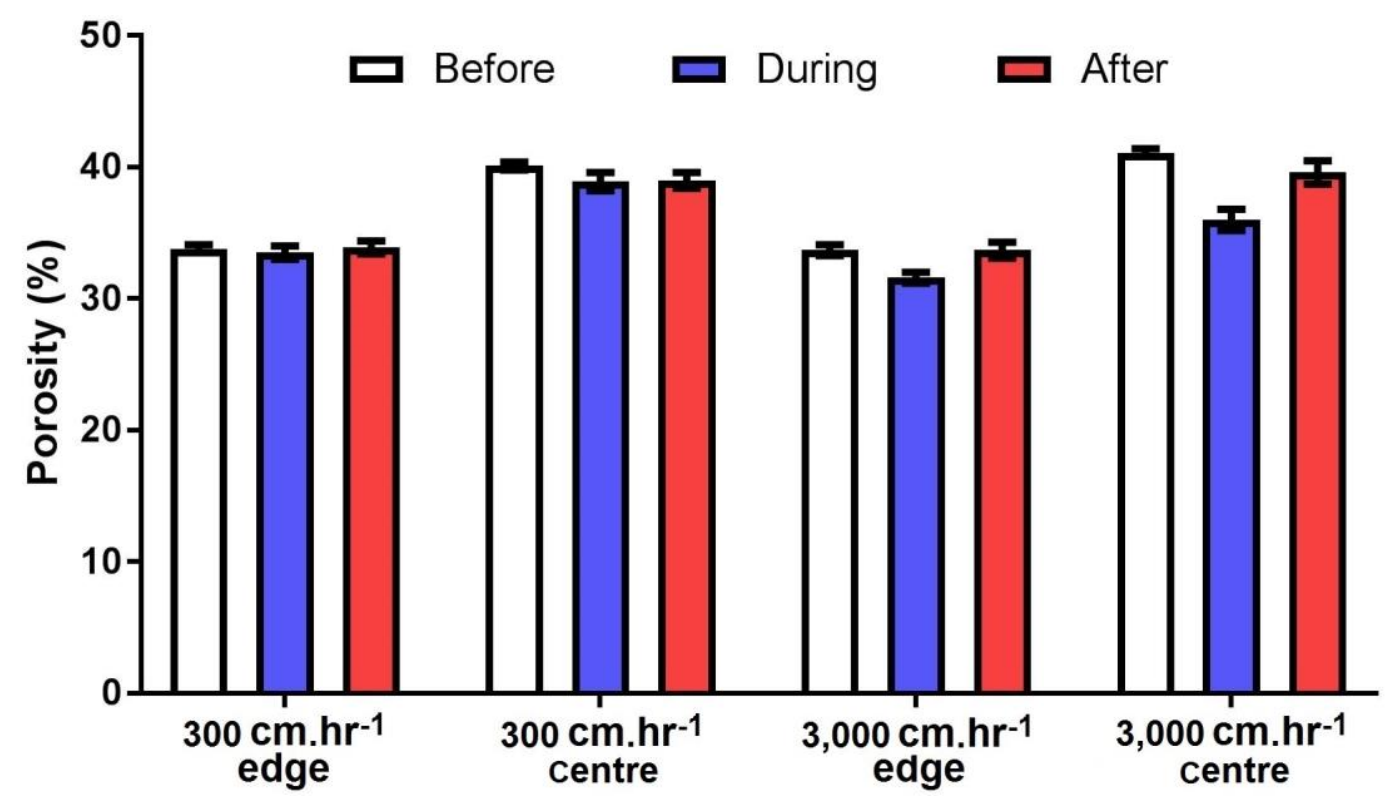

B

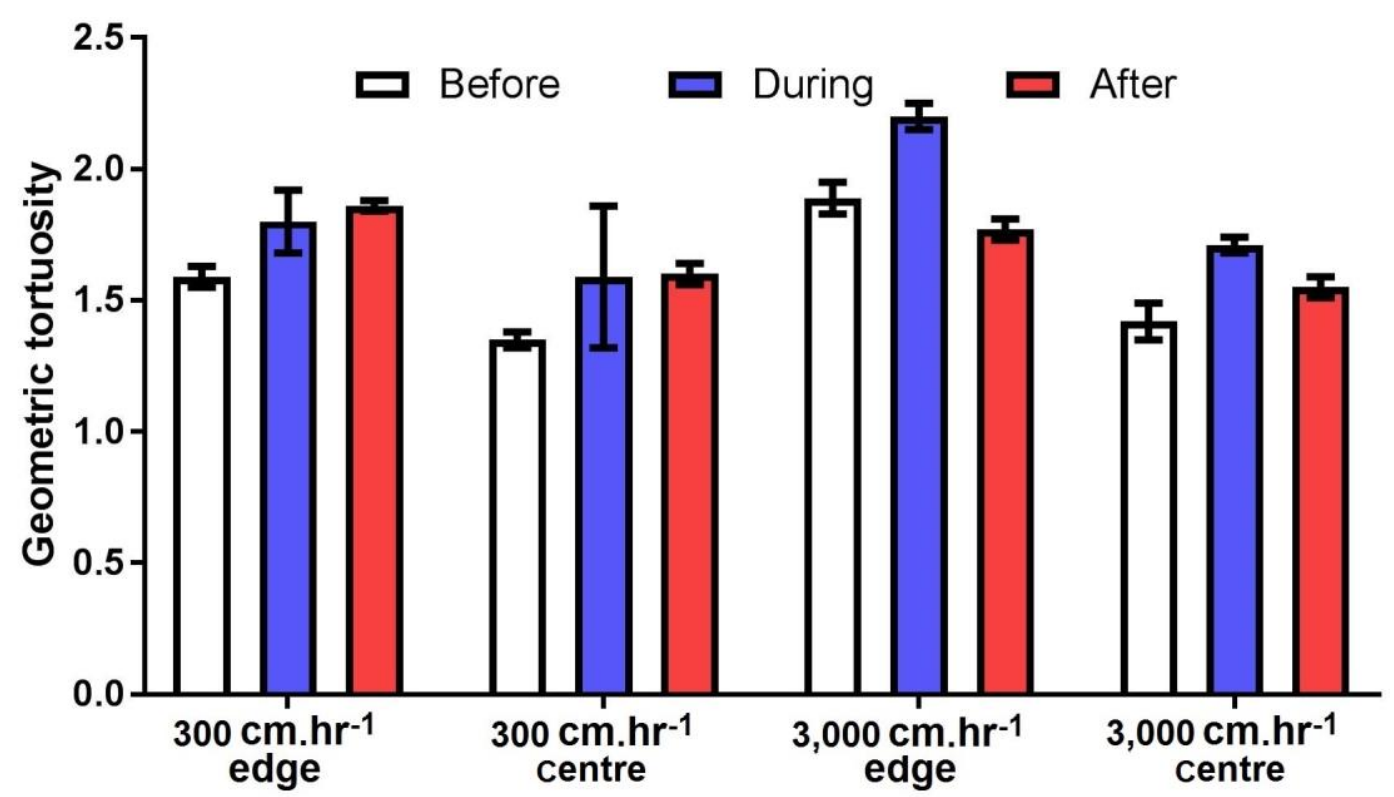


C

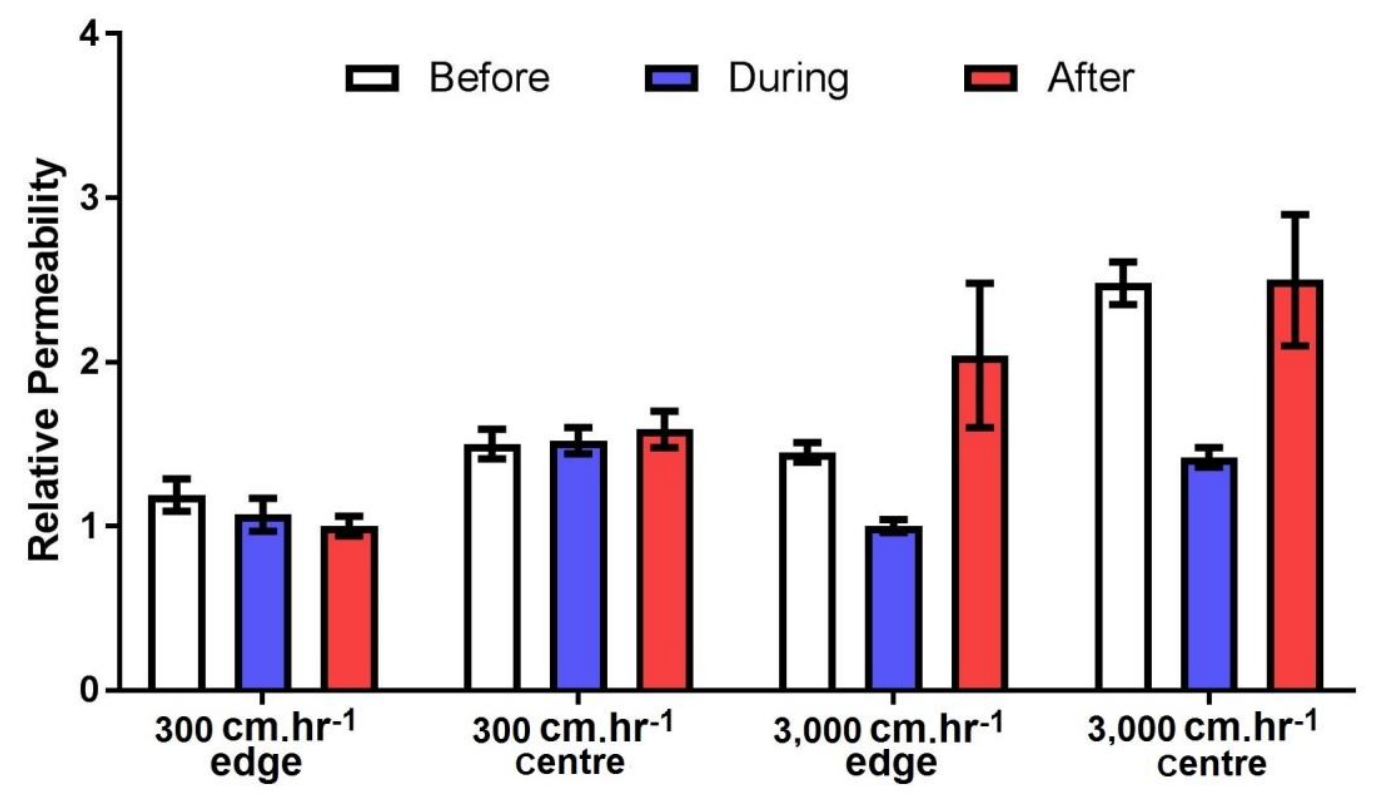

Figure 5

A

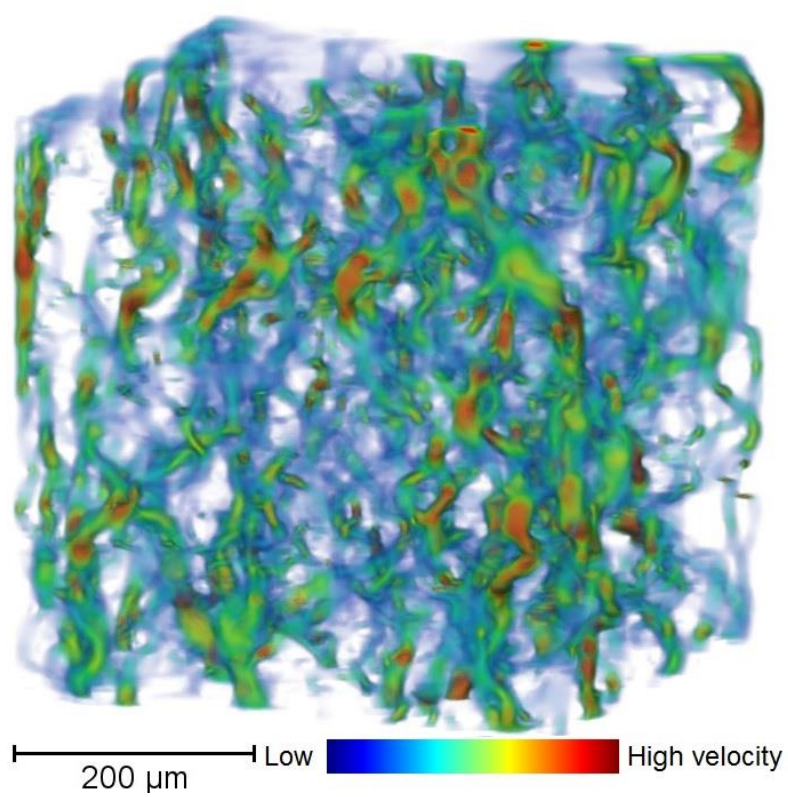


B

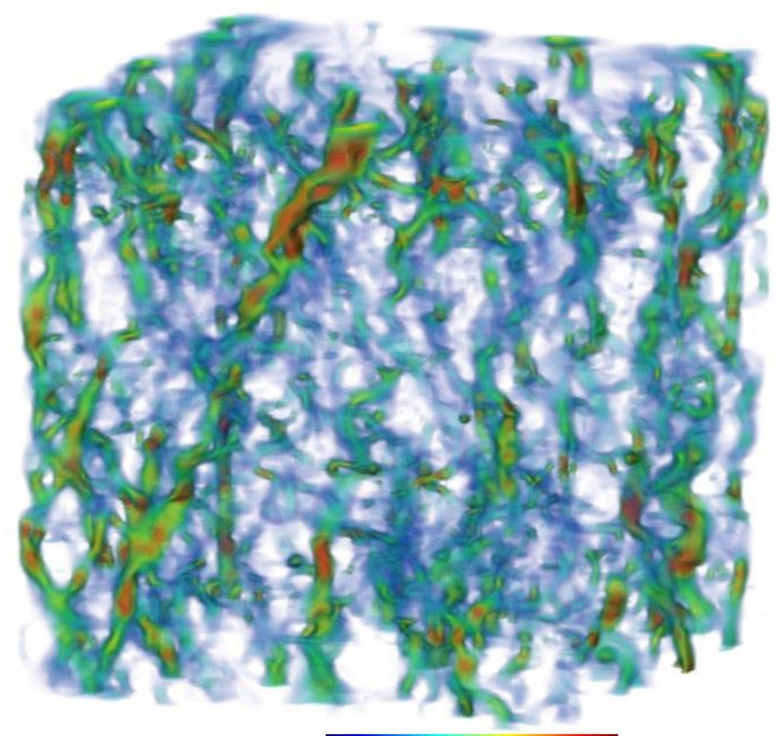

$200 \mu \mathrm{m}$

High velocity

\section{Figure 6}

\section{A}

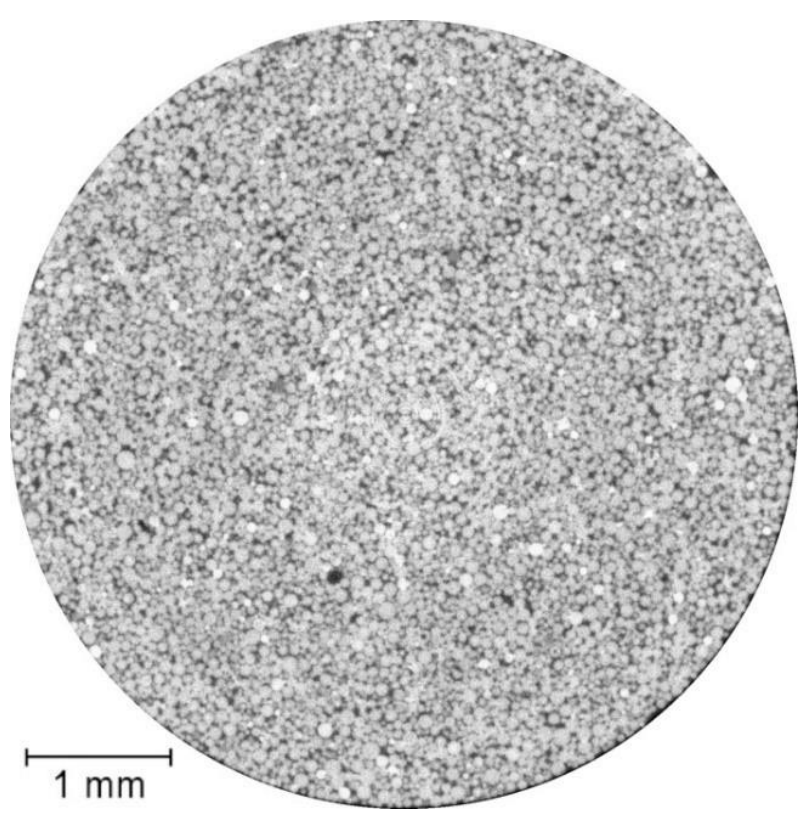




\section{B}

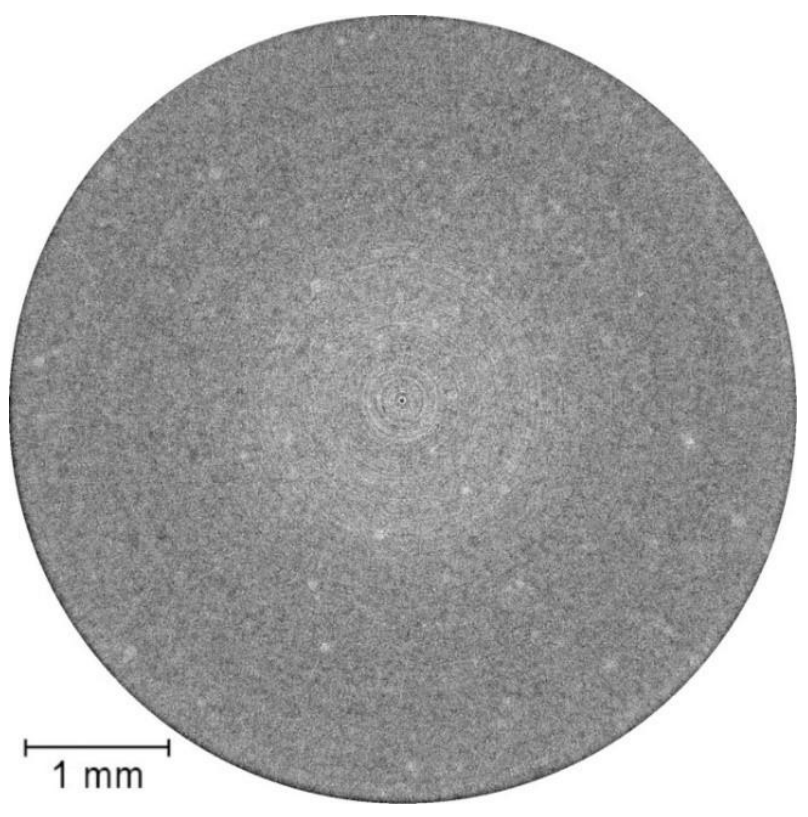

Figure 7

A

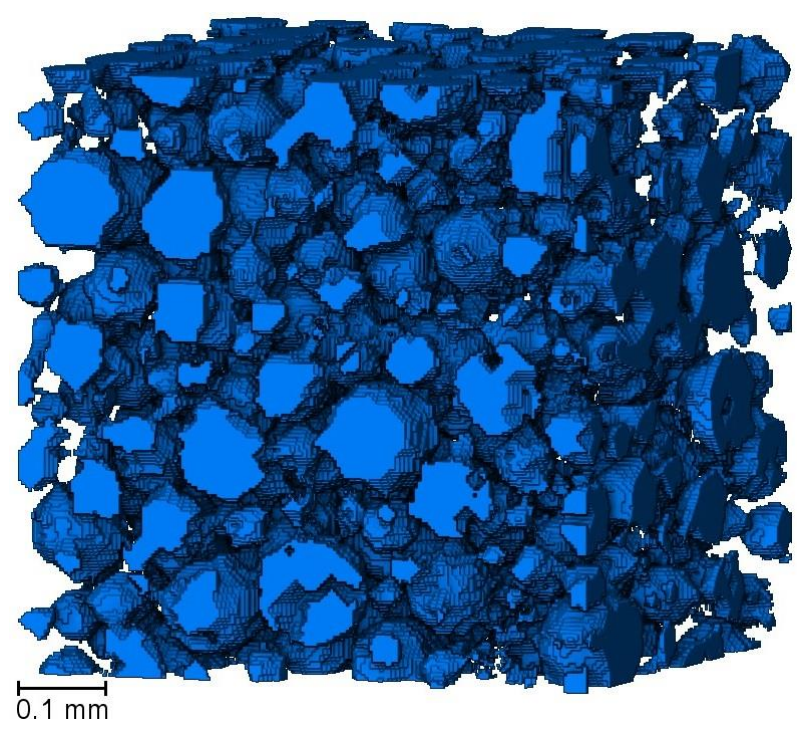

Page $\mathbf{2 4}$ of $\mathbf{2 8}$ 
B

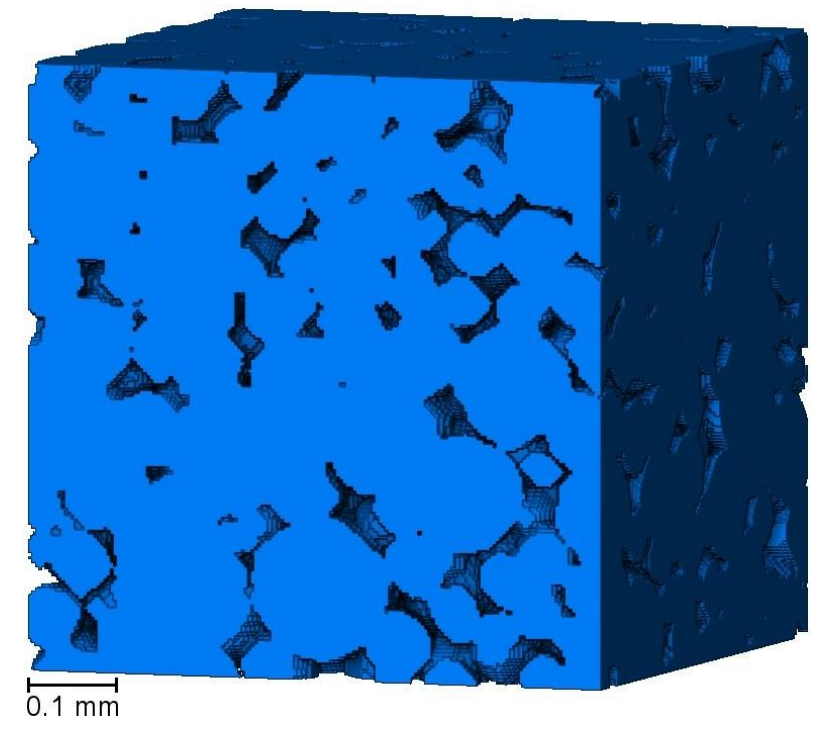

C

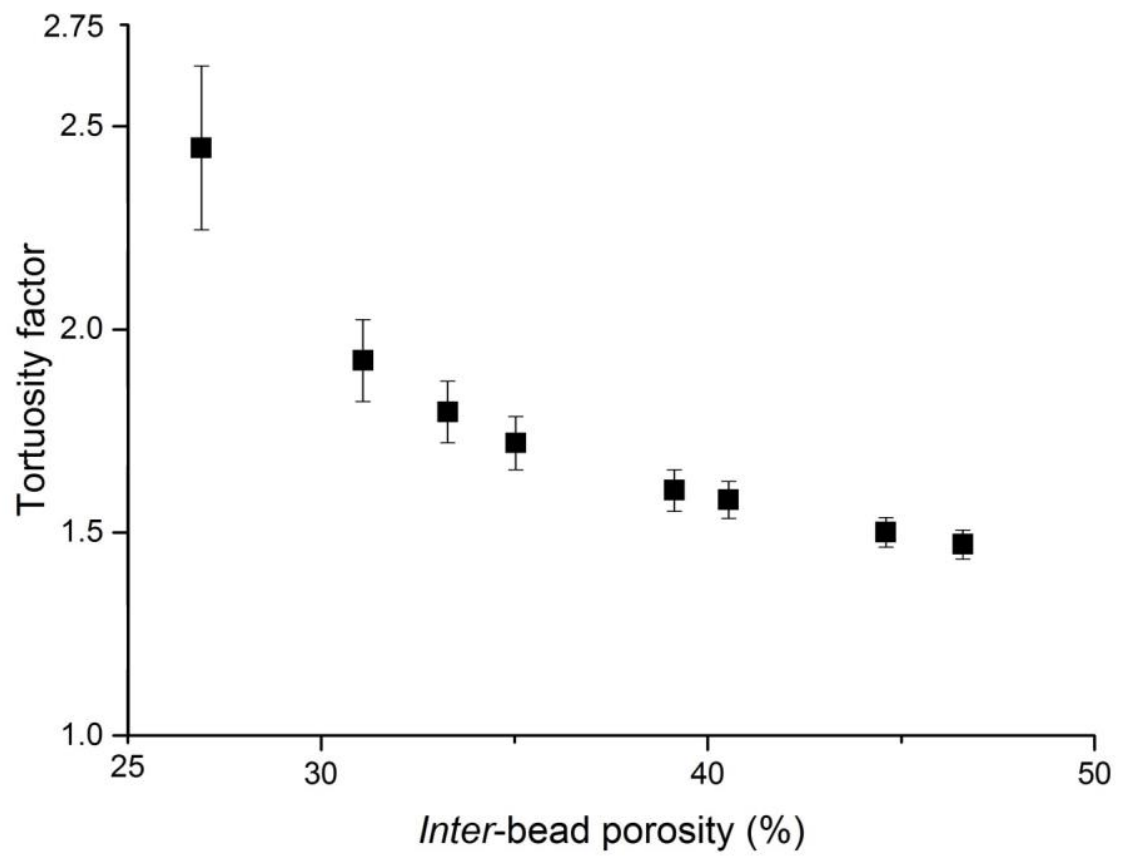


Figure 8

A

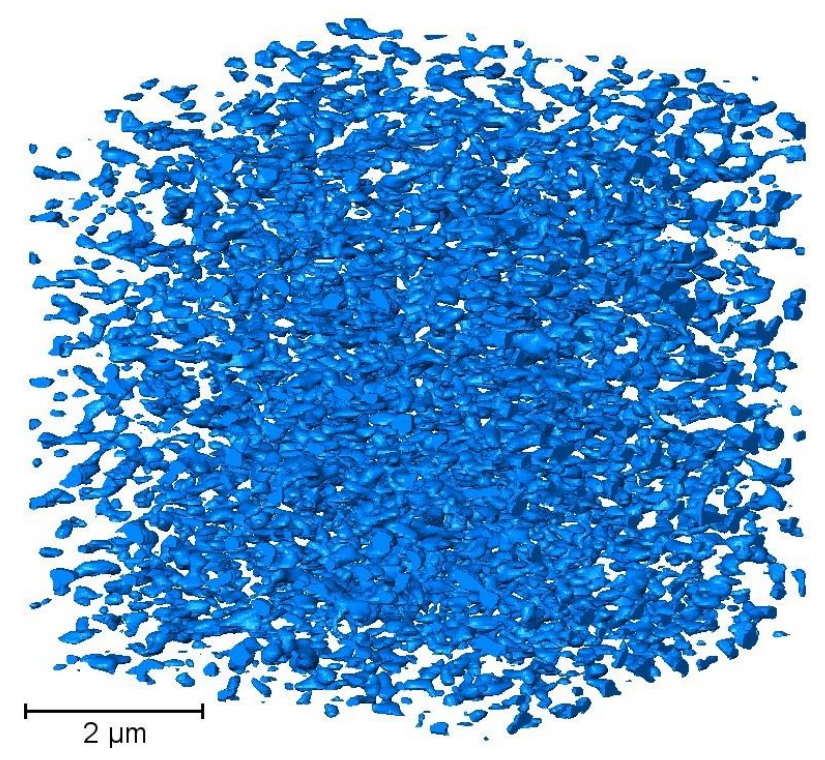

B

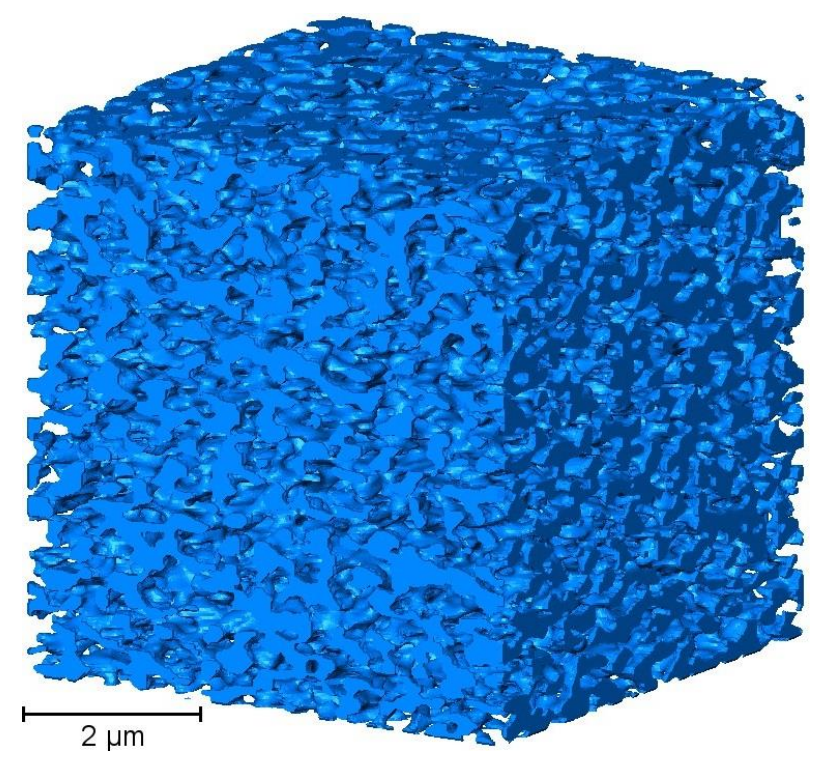


C

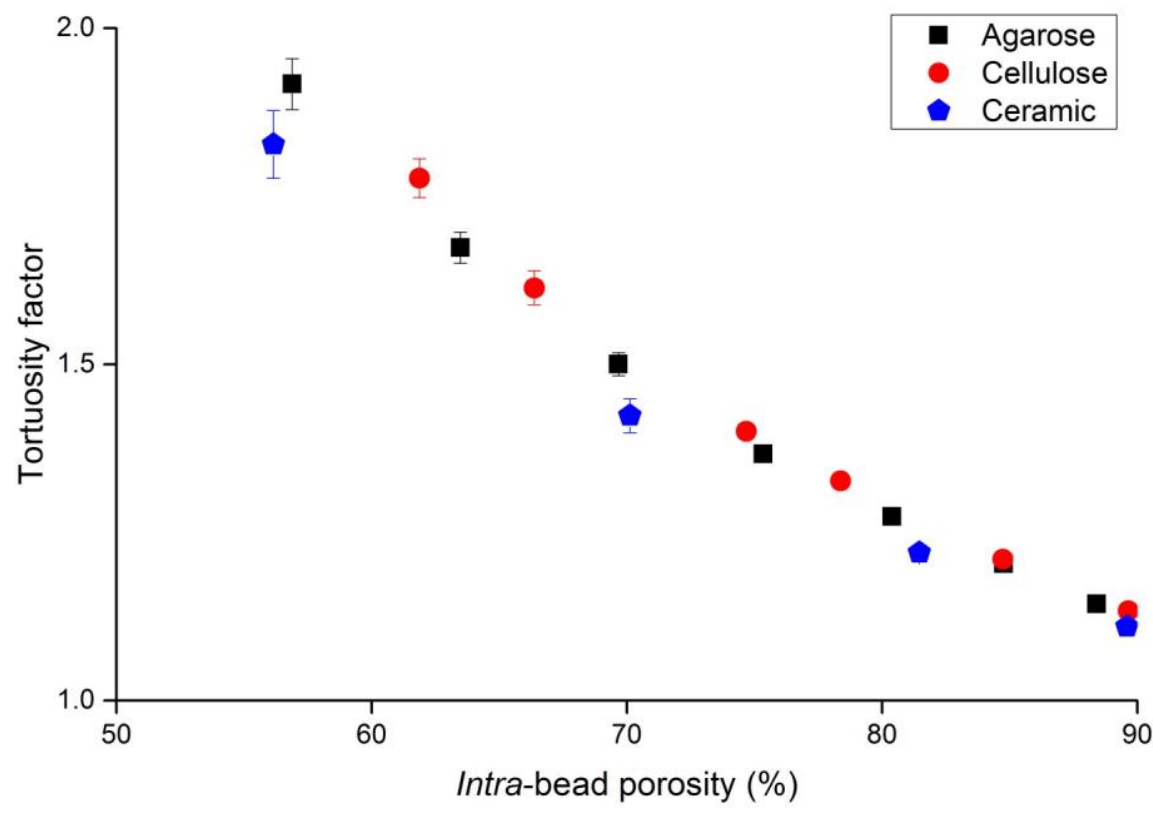




\section{Table 1}

\begin{tabular}{|c|c|c|c|c|c|c|}
\hline $300 \mathrm{~cm} \cdot \mathrm{h}^{-1}$ & \multicolumn{3}{|c|}{ Edge } & \multicolumn{3}{|c|}{ Centre } \\
\hline \multirow{3}{*}{ Geometric porosity (\%) } & Before & During & After & Before & During & After \\
\hline & 33.8 & 33.5 & 33.9 & 40.1 & 38.9 & 39.0 \\
\hline & \pm 0.3 & \pm 0.5 & \pm 0.5 & \pm 0.3 & \pm 0.7 & \pm 0.6 \\
\hline \multirow{2}{*}{ Geometric tortuosity } & 1.59 & 1.80 & 1.86 & 1.35 & 1.59 & 1.60 \\
\hline & \pm 0.04 & \pm 0.12 & \pm 0.02 & \pm 0.03 & \pm 0.27 & \pm 0.04 \\
\hline \multirow{2}{*}{ Relative permeability } & 1.00 & 0.89 & 0.84 & 1.26 & 1.27 & 1.33 \\
\hline & \pm 0.09 & \pm 0.08 & \pm 0.05 & \pm 0.08 & \pm 0.07 & \pm 0.09 \\
\hline \multirow{2}{*}{ Average pore diameter $(\mu \mathrm{m})$} & 11.8 & 11.3 & 11.1 & 11.9 & 11.5 & 11.5 \\
\hline & \pm 0.0 & \pm 0.6 & \pm 0.0 & \pm 0.1 & \pm 0.5 & \pm 0.1 \\
\hline \multirow[t]{2}{*}{$3,000 \mathrm{~cm} \cdot \mathrm{h}^{-1}$} & \multicolumn{3}{|c|}{ Edge } & \multicolumn{3}{|c|}{ Centre } \\
\hline & Before & During & After & Before & During & After \\
\hline \multirow{2}{*}{ Geometric porosity (\%) } & 33.7 & 31.6 & 33.7 & 41.1 & 36.0 & 39.6 \\
\hline & \pm 0.4 & \pm 0.4 & \pm 0.6 & \pm 0.3 & \pm 0.8 & \pm 0.9 \\
\hline \multirow{2}{*}{ Geometric tortuosity } & 1.89 & 2.20 & 1.77 & 1.42 & 1.71 & 1.55 \\
\hline & \pm 0.06 & \pm 0.05 & \pm 0.04 & \pm 0.07 & \pm 0.03 & \pm 0.04 \\
\hline \multirow{2}{*}{ Relative permeability } & 1.00 & 0.69 & 1.40 & 1.71 & 0.98 & 1.72 \\
\hline & \pm 0.06 & \pm 0.03 & \pm 0.30 & \pm 0.09 & \pm 0.04 & \pm 0.28 \\
\hline \multirow{2}{*}{ Average pore diameter $(\mu \mathrm{m})$} & 9.1 & 8.1 & 9.4 & 10.5 & 8.8 & 9.9 \\
\hline & \pm 0.8 & \pm 0.1 & \pm 0.0 & \pm 0.6 & \pm 0.1 & \pm 0.1 \\
\hline
\end{tabular}

\section{Table 2}

\begin{tabular}{c|ccc}
$\begin{array}{c}\text { Relative } \\
\text { porosity }\end{array}$ & \multicolumn{2}{c}{ Diffusivity coefficient $\left(\mathbf{m}^{2} . \mathbf{s}^{-1}\right),\{$ Geometric porosity (\%)\} } \\
\cline { 2 - 4 } & Agarose & Cellulose & Ceramic \\
High & $(9.4 \pm 0.2) \times 10^{-12},\{85\}$ & $(6.8 \pm 0.0) \times 10^{-12},\{85\}$ & $(1.1 \pm 0.0) \times 10^{-11},\{82\}$ \\
Medium & $(3.9 \pm 0.2) \times 10^{-12},\{70\}$ & $(2.2 \pm 0.2) \times 10^{-12},\{75\}$ & $(4.1 \pm 0.2) \times 10^{-12},\{70\}$ \\
Low & $(5.2 \pm 0.7) \times 10^{-13},\{57\}$ & $(3.2 \pm 1.4) \times 10^{-13},\{62\}$ & $(1.1 \pm 0.1) \times 10^{-12},\{56\}$ \\
\hline Literature & $\left(7.8\right.$ to 8.0) $\times 10^{-12}[41]$ & $\left(1.9\right.$ to 12) $\times 10^{-12}[42]$ & $\left(3.6\right.$ to 4.7) $\times 10^{-11 *}[43]$
\end{tabular}

